\title{
Prioritizing habitat patches for conservation in fragmented landscapes/townscapes using network-based models and analyses
}

\author{
Ö. Bodin \\ Stockholm Resilience Centre \& Dept. of Systems Ecology, \\ Stockholm University, Sweden
}

\begin{abstract}
To preserve biodiversity in highly fragmented landscapes, it is crucial to conserve and possibly improve the connectivity among remaining habitat patches. However, multiple users are competing for a limited amount of land, and conservation efforts subsequently need to be efficiently directed to maximize biodiversity given limited resources. In terms of connectivity, this could be expressed as to which habitat patches do we really need to preserve, and which patches could we lose without facing any significant negative effects on connectivity? Network-based models of fragmented landscapes provide for comprehensive visualizations and analyses of landscape connectivity that could help in prioritizing habitat patches for conservation. This is especially valuable in a planning context where many different types of agents are typically involved, thus emphasizing the importance of being able to present key ecological implications of different spatially explicit habitat configurations in a easily understandable way.

Here, three different aspects of landscape connectivity are presented, all suggested as being particularly suitable for network-based modeling approaches. These are: (i) estimating to what degree the landscapes spatial configuration enables re-colonization following local extinctions; (ii) identifying clusters of patches that together form sufficient habitat; and (iii) identifying key-stone patches that are crucial in providing connectivity.
\end{abstract}

Keywords: habitat patches, land use planning, network models, landscape fragmentation, graphs. 


\section{Introduction}

Biodiversity and species persistence is highly dependent on access to high quality habitats. Societal and economic development, on the other hand, often leads to the reduction of both the amount and the quality of natural habitats. The amount of natural habitat decreases as more land is converted to, for example, agricultural production or housing development. Also, the habitat quality can be reduced when, for example, a previously relatively undisturbed forest fragment is converted to modern monoculture forestry, or to a recreational park. In addition, the reduction of natural habitat increases the level of fragmentation among remnant habitat patches. A patch represents a spatially contiguous area of land with a biophysical composition which potentially suits as adequate habitat for a focal species. An increased level of fragmentation implies that the connectivity among remnant habitat patches is decreased. Connectivity is, on a general level, the degree to which the spatial pattern of habitat patches in the landscape facilitates or impedes the movement of organisms (Taylor et al [1]). Decreased possibilities for species movements can, for example, result in local species extinctions (Levin [2]) and thus in biodiversity reductions. Hence, it is not only the biophysical composition (i.e. the quality) and the geographical area of a given habitat patch as such that define its ecological value, but its level of connectivity in relation to other habitat patches in a landscape is also important.

An important arena for decision-making about various land uses that affects the amount, quality and connectivity of species habitat are the planning processes at the municipality level. Here, as well as in many other contexts where different usages of land are decided, multiple users with different interests, knowledge and perceptions are competing for a limited amount of land. In such settings, conservation efforts subsequently need to be efficiently directed to maximize biodiversity given limited resources. Since land uses are decided in a competitive context where different interests are to be weighted against each other in the planning process, tools that provide the various decision-makers and stakeholders with adequate assessment of the ecological importance of different habitat patches are crucial. This kind of tool would help in making informed decisions on which habitat patches to preserve in maximizing various ecological values, given that there are other competing land uses, and which patches could be converted to other purposes without any severe ecological consequences.

Furthermore, it is not only the sheer existence of such tools that is important. Firstly, the actors that, to some degree, represent the interest of preserving/creating habitat would benefit from being able to use the tools themselves without the need for constant support from external experts. This is in part a result of the dynamic nature of the planning process itself where different scenarios of land uses are constantly being presented and contested. In such situations, it is highly beneficial if the conservation agents have access to tools that they can use themselves to evaluate different land use scenarios more or less in real time instead of having to solely rely on assessments conducted by external experts on a consultation basis. Therefore, ease of use is of central value for any such tool. Secondly, it is important that these tools provide results that 
are fairly easily comprehensible by non-experts and lay men. If the results from ecological assessment are hard to understand and interpret, it might be hard to gain acceptance and support for different habitat conservation schemes among other actors involved in the planning process.

A fairly recent innovation in studying various aspects of landscape connectivity resulting from habitat fragmentation is the network-centric modeling approach (Keitt et al [3], Cantwell et al [4], Urban and Keitt [5]). This modeling approach (which is often called graph-theoretical) has also been specifically suggested as very useful in prioritizing and ranking the importance of different habitat patches from a connectivity perspective e.g. Pascual-Hortal and Saura [6]. Furthermore, the modeling approach as such does not rely on large quantities of detailed ecological data; rather it can quickly provide a coarsegrain analysis of an entire landscape using fairly low amounts of data. The network modeling approach thus provides for a favorable trade-off between how well the model portrays reality and the data it requires to do so (Calabrese and Fagan [7]). Hence, this modeling approach fulfils the different aspects suggested as important for an effective patch prioritizing tool for use in planning as presented here, and is therefore a promising candidate for further development and possibly also for deployment in real-world planning situations.

In this work we will further examine the potential of the network modeling approach by specifically studying how it can be used to study three important and different, although not mutually exclusive, aspects of landscapes connectivity. These aspects are: (1) large-scale connectivity which is important, for example, in metapopulation dynamics; (2) local connectivity, which is important in order to provide sufficient home ranges in highly fragmented areas; and (3) identification of critical patches that much more than others provide for critical connections and dispersal pathways in the landscape (e.g. stepping stones). The study is based on a review of a selected sample of the emerging literature applying network based modeling in studying landscape ecology and/or metapopulation dynamics.

\section{The network model of landscapes}

The network modeling approach rests on the basic abstraction of a landscape as consisting of scattered patches of habitat that are laid out in a landscape matrix which, to a varying degree, is inhospitable for the focal species, and that the connectivity between any pair of habitat patches depends on the effective distance separating them. If the effective distance is below the maximum dispersal distance of the focal species, that particular pair of patches is considered as connected in terms of possibilities for direct dispersal. In network terminology, habitat patches are the nodes in the network, and all pairs of patches that are separated by an effective distance below the maximum dispersal distance of the focal species are connected by links (Fig. 1).

The effective distance is dependent on the geographical distance separating the patches and the permeability of the different types of land making up the matrix between the different patches. If the matrix is very hard to cross, either 
from an energetic and/or risk of mortality due to the predation perspective, the permeability is considered low, and two patches geographically close to each other would be modeled as separated by a longer effective distance. In a GIS, cost-distance analyses can be applied to calculate the effective distances separating patches e.g. Bunn et al [8], Verbeylen et al [9].
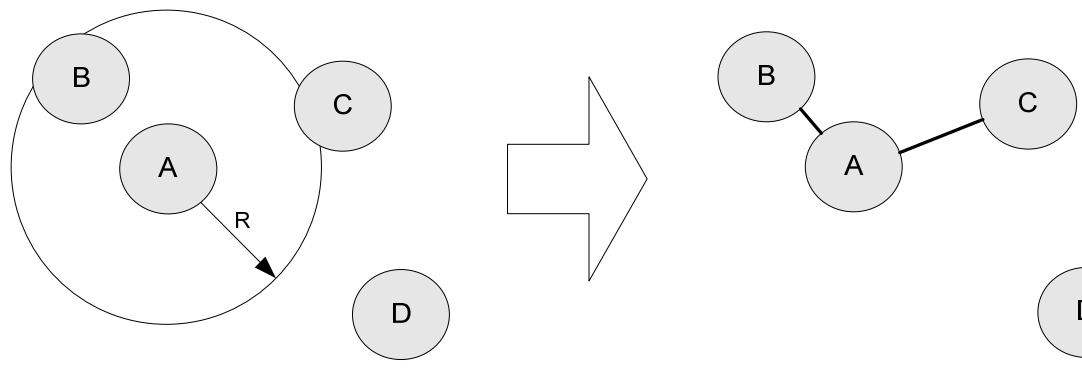

Figure 1: The habitat patches A-D are located in the landscape, and $\mathrm{R}$ is the maximum dispersal distance of the focal species. All patches separated with a distance less than $\mathrm{R}$ are considered as connected (right side).

Furthermore, a central assumption is that a species can disperse along paths consisting of several links. Thus, species are assumed to be able to move between patches that are not directly, but indirectly connected through network paths (e.g. from $\mathrm{B}$ to $\mathrm{C}$ via $\mathrm{A}$ in Fig. 1). Hence, a species would be able to disperse throughout large areas of the landscape by moving from patch to patch (assuming that such paths exist). In other words, the network model merges species dispersal processes with spatial patterns of habitat patches in one single model.

The network model of the fragmented landscape provides, however, only for the basic data structure which can be further analyzed using various methods and metrics from research fields such as social network analysis and/or graph theory (Bodin and Norberg [10]). This fairly simple but still relevant abstraction of the complex patterns of connectivity among habitat patches makes connectivity analyses of fragmented landscapes easier to conduct, and many more or less standardized and easily assessable tools and methods are available off-the-shelf (see e.g. Bodin and Norberg [10]).

\section{Three ecological aspects related to landscape fragmentation}

\subsection{Overall connectivity and species persistence}

Several decades ago, Levin [2] introduced the concept of metapopulation. A metapopulation is a set of spatially separated local populations which may, individually, undergo local extinctions, but where the aggregated population is maintained by re-colonization events resulting from dispersing organisms. 
Hence, upholding a high level of landscape connectivity is central in order to provide for enough dispersion to prevent overall population declines induced by the occurrence of local extinctions. Building on earlier work, Hanski and Ovaskainen [11] introduced a spatially explicit metapopulation model that incorporates the flux of dispersing organisms between individual patches at the landscape level. Their model uses spatially explicit information from a real landscape to access the capacity of a specific metapopulation to actually persist in that particular landscape. This research shows, for example, that metapopulation dynamics is very important to take into account when designing natural reserves. A key design issue is therefore to make sure that the level of connectivity among reserves is high enough given species dispersal capabilities (e.g. Moilanen and Cabeza [12]). If reserves are too geographically separated, local extinctions are to be expected (Cabeza and Moilanen [13]).

For this kind of study and assessment, network-based models of landscape have great potential (alongside other modeling approaches). Here, the possible existence of network components is of key interest. A network component is an isolated subnet confined within a larger network. By definition, no links exist between nodes of different components. Thus, if a network model describing a particular landscape consists of two or more network components, a dispersing species cannot traverse the entire landscape in moving from patch to patch (Keitt et al [3], Urban and Keitt [5]). The ecological interpretation of a network consisting of several network components is that species can only disperse to patches belonging to the same component, and are thereby isolated from other network components in the landscape. Thus, a network component would correspond to a single metapopulation which in turn may consist of one or several individual local populations confined to patches within that particular network component. If a network component is small, the entire metapopulation would be small only consisting of a very limited number and/or sizes of local populations and, therefore, far less persistent than a larger metapopulation. From a conservation perspective, it would consequently be important to aim for a spatial configuration of the habitat patches in the landscape that would minimize the number of network components. This is in line with findings derived using other modeling approaches which have led to the suggestion that natural reserves should be clustered together spatially (Cabeza et al [14]).

Several other analyses building on, or extending, the component based network analysis have been suggested. As a consequence of the binary perspective of component based analysis, the ability to estimate a more continual degree of spatial separation of the patches in the landscape is reduced. This is apparent in cases where different areas of the landscape are not necessarily completely isolated from each other, but where patches are confined to different spatially distinguishable clusters. Bodin and Norberg [10] applied a networkbased method called Community structure (Girvan and Newman [15]) to assess different spatially distinguishable groups of patches that were not necessarily complete isolated from each other. This method is suggested as particularly suitable in landscapes where species' dispersals is not fully inhibited by fragmentation, but merely limited. 
Furthermore, network-based analytical methods do not take characteristic of the nodes (i.e. the patches) into account. In studying landscapes, that presents a possible limitation if the area and the quality of the patches, in addition to their connectivity, are to be taken into account (see e.g. Ferrari et al [16]). In order to address that limitation, Pascual-Hortal and Saura [6] introduced the metric Integral index of connectivity (IIC) which do not only consider the number of network component, but also the area of the patches within the components. This approach, which they termed habitat availability, integrates habitat amount and connectivity in one single metric. This metric was later extended to account for varying probabilities for dispersals between different pair of patches (Saura and Pascual-Hortal [17]).

\subsection{Providing home range}

Metapopulations processes are occurring on the level of populations, and are characterized by dynamics spanning several generations in time. On the other hand, day-to-day movements of individuals are taking place on much smaller spatial and temporal scales. The extent of land covered during the course of species everyday activities, i.e. their home ranges, is very limited in comparison with the areas covered by species populations. However, habitat fragmentation can be a serious problem even on these smaller scales. If not sufficient amount of habitat is easily accessible given a species propensity for daily movements, that particular locality might very well be left unoccupied irrespectively of the potential existence of any nearby species populations. This is often a problem is severely fragmented landscapes such as urban areas (Andersson and Bodin [18]). Similarly as when studying metapopulations, the connectivity among remnant habitat patches defines to what extent a particular locality may or may not provide an adequate home range for a focal species. Although the spatial scale, and the ecological problem at hand, is quite different, the network-based analysis is very similar. The differences are (i) the significantly lower dispersal capacity of the focal species for daily movement in comparison with its capacity for natal dispersal, and (ii) the species habitat area requirement. The latter relates to the species daily needs in terms of access to sufficient habitat for foraging and shelter.

A recent study of the presence/absence of bird species in an urban context applied the previously mentioned network component based analysis to empirically test the modeling approach as such. The study also estimated, for some species, the maximally allowed distance between patches within a home range, and the lowest acceptable amount of habitat area accessible within the home ranges (Andersson and Bodin [18]). The results are encouraging in terms of the network models predictive capacity, and therefore provide scientifically based support for network based models as such.

Another analytical approach that can be applied to identify areas within a landscape that can provide sufficient home range is to look at different measures of network centrality. Centrality measures are used to assess the impact, influence or prominence of individual nodes based on their position in the network (see e.g. Freeman [19] for a overview of how centrality measures can be 
applied in studying social networks). By investigating the ecological applicability of several different measures of network centrality, a recent study suggests that groups of patches with high scores on the subgraph and closeness centralities tend to be clustered together therefore forming islands within the landscape with particularly high internal connectivity (Ernesto et al [20]). In a very fragmented landscape, these high-connectivity islands might make up the only localities where species with limited daily movement capabilities can actually thrive.

\subsection{Critical habitat patches}

In real landscapes, habitat patches are seldom uniformly separated in space. Thus, irrespectively of whether focus is on studying spatial configurations of habitat patches in relation to metapopulation dynamics or the provisioning of adequate home ranges, some patches are likely to be more important than other in terms of their individual contribution to these different aspects of connectivity. The flip side of the coin is that other patches will be of less importance. Network-based methods can be applied to assess and rank habitat patch importance using, broadly defined, two different approaches. The first approach uses a two stage process where the first step is to choose a specific network metric of interest given the problem at hand (which could, for example, be the number of network component in a particular landscape). Then, each individual patch is removed, one at the time, from the network model and the resulting effect on the chosen metric is recorded (e.g. Urban and Keitt [5], Pascual-Hortal and Saura [6]). Individual patches are then ranked according to how much the chosen connectivity metric decreased following their removal. Hence, this approach is experimental, although the experiments are purely theoretical. The second approach is to define network measures that are thought to capture some aspects of importance regarding individual patches' contribution to the connectivity. The previously mentioned concept of network centrality is here of focal interest (e.g. Bodin and Norberg [10], Ernesto and Bodin [20], Minor and Urban [21]). Although this approach is inherently dependent on an appropriate and relevant ecological interpretation of any network-centric centrality metric, it has the benefit of, to some degree, being able to assess patch importance in removal scenarios where more than one patch is lost. The former experimental approach, on the other hand, which carries the advantage of a relatively straightforward ecological interpretation, instead inherently depends on some often arbitrary chosen patch removal sequences in those cases where more than one patch is lost.

Both approaches can, for example, be used to assess how much a patch contributes to (i) upholding the long-range connectivity (or traversability) of the entire network from a topological perspective, and (ii) function as a source for dispersing organisms. The latter relates to the area of the patch in combination with the magnitude of its outgoing fluxes of organisms (see e.g. Minor and Urban [21]). Source areas are clearly important as providers of dispersing organisms that can re-colonize patches following local extinctions, and/or as source areas which enable the presence of species in other nearby patches that 
otherwise would have been unoccupied. An appropriate metric could, for example, be the change in the previously mentioned metric IIC following experimental patch removals (Pascual-Hortal and Saura [6]). The former topologically oriented patch ranking assessment is related to how much a patch provides for long-range connectivity between distant patches and is proposed to be captured by the network centrality measure of betweenness (Bodin and Norberg [10]). Betweenness centrality has also, in a conceptually similar way, recently been applied in assessing and ranking subpopulations in terms of their impact in relaying gene flow or sustaining a metapopulation system (Rozenfeld et al [22]).

\section{Discussion}

The level of connectivity is often severely restrained in highly fragmented areas such as in urbanized landscapes. To preserve and improve biodiversity in such settings, it is crucial to conserve and possibly improves the connectivity among remaining habitat patches. The three ecological issues of importance presented here (metapopulation dynamics, accessible home ranges, individual habitat patch criticality), which all relate to the spatial configuration of habitat patches in fragmented landscape, presents different challenges for land use planning and conservation. Regarding the first issue, network-based analyses could be used to assess whether a landscape would be capable of harboring one or several metapopulations, and to some degree assess the capacity of the landscape to sustain these metapopulations over time. The modeling approach would also be able to identify where such metapopulations would be spatially confined, thereby defining ecologically relevant geographical sub-regions of the landscape. Since such ecologically defined regional subdivisions of the landscape will rarely coincide with juridical borders among different governing agencies, crossboundary management approaches might be preferable in these cases (see e.g. Borgstrom et al [23]).

Also, network-based modeling approaches can be used to identify local cluster of patches (cf. home ranges) that can harbor at least a small species population, thus identifying areas within the landscape that might be worth protecting from further habitat reduction. From another angle, these identified high-density clusters could also be seen as areas there the amount of habitat could be reduced while still minimizing any negative ecological consequences, given that the focal species is capable of withstanding its presence in spite of the induced loss in habitat connectivity.

Furthermore, a key challenge for effective conservation in planning situations is to be able to assess the importance of individual habitat patches. In doing so, conservation efforts can be focused to preserve those specific patches whose removal would do most harm from an ecological point of view. A range of network-based metrics has, as an attempt to meet this challenge, been proposed to assess and rank individual patches in terms of their different contribution to various aspects of landscape connectivity (such as the connectivity aspects 
described in this study). Hence, analysis of individual patch importance is part of the portfolio of analyses made possible using the network modeling approach.

Finally, in addition to the scientific potentials of the network-based modeling approach in analyzing these key ecological issues of importance, the model itself and the outcomes of the model are also fairly easy to interpret and understand by non-experts. The latter is suggested as being of particular importance in planning situation where a multitude of different agencies and stakeholders are typically involved.

\section{Acknowledgements}

This research was supported by the Swedish Research Council for Environment, Agricultural Sciences and Spatial Planning (Formas), and the Foundation for Strategic Environmental Research (Mistra).

\section{References}

[1] Taylor P. D., Fahrig L., Henein K. \& Merriam G., Connectivity is a vital element of landscape structure. Oikos, 68, pp. 571-573, 1993.

[2] Levin R., Some Demographic and Genetic Consequences of Environmental Heterogeneity for Biological Control. Bulletin of the Entomological Society of America, 15, pp. 237-240, 1969.

[3] Keitt T. H., Urban D. L. \& Milne B. T., Detecting Critical Scales in Fragmented Landscapes. Conservation Ecology, 1(1), pp. 1997.

[4] Cantwell M. D. \& Forman R. T. T., Landscape Graphs - Ecological Modeling with Graph-Theory to Detect Configurations Common to Diverse Landscapes. Landscape Ecology, 8(4), pp. 239-255, 1993.

[5] Urban D. \& Keitt T., Landscape connectivity: A graph-theoretic perspective. Ecology, 82(5), pp. 1205-1218, 2001.

[6] Pascual-Hortal L. \& Saura S., Comparison and development of new graphbased landscape connectivity indices: towards the prioritization of habitat patches and corridors for conservation. Landscape Ecology, 21(7), pp. 959967, 2006.

[7] Calabrese J. M. \& Fagan W. F., A comparison-shopper's guide to connectivity metrics. Frontiers in Ecology and the Environment, 2(10), pp. 529-536, 2004.

[8] Bunn A. G., Urban D. L. \& Keitt T. H., Landscape connectivity: A conservation application of graph theory. Journal of Environmental Management, 59(4), pp. 265-278, 2000.

[9] Verbeylen G., Bruyn L. D., Adriaensen F. \& Matthysen E., Does matrix resistance influence Red squirrel (Sciurus vulgaris L. 1758) distribution in an urban landscape? Landscape Ecology, 18, pp. 791-805, 2003.

[10] Bodin Ö. \& Norberg J., A Network Approach for Analyzing Spatially Structured Populations in Fragmented Landscape. Landscape Ecology, 22(1), pp. 31-44, 2007. 
[11] Hanski I. \& Ovaskainen O., The metapopulation capacity of a fragmented landscape. Nature, 404, pp. 755-758, 2000.

[12] Moilanen A. \& Cabeza M., Single-species dynamic site selection. Ecological Applications, 12(3), pp. 913-926, 2002.

[13] Cabeza M. \& Moilanen A., Site-selection algorithms and habitat loss. Conservation Biology, 17(5), pp. 1402-1413, 2003.

[14] Cabeza M., Araujo M. B., Wilson R. J., Thomas C. D., Cowley M. J. R. \& Moilanen A., Combining probabilities of occurrence with spatial reserve design. Journal of Applied Ecology, 41(2), pp. 252-262, 2004.

[15] Girvan M. \& Newman M. E. J., Community structure in social and biological networks. Proceedings of the National Academy of Sciences of the United States of America, 99(12), pp. 7821-7826, 2002.

[16] Ferrari J. R., Lookingbill T. R. \& Neel M. C., Two measures of landscapegraph connectivity: assessment across gradients in area and configuration. Landscape Ecology, 22, pp. 1315-1323, 2007.

[17] Saura S. \& Pascual-Hortal L., A new habitat availability index to integrate connectivity in landscape conservation planning: Comparison with existing indices and application to a case study. Landscape and Urban Planning, 83, pp. 91-103, 2007.

[18] Andersson E. \& Bodin Ö. Practical tool for landscape planning? An empirical investigation of network based models of habitat fragmentation. Ecography, Published online (early view), 2008.

[19] Freeman L., Centrality in social networks. Conceptual clarifications. Social Networks, 1, pp. 215-239, 1979.

[20] Ernesto E. \& Bodin Ö. Using network centrality measures to manage landscape connectivity. Ecological Applications, 18(7), pp. 1810-1825, 2008.

[21] Minor E. S. \& Urban D. L., Graph theory as a proxy for spatially explicit population models in conservation planning. Ecological Applications, 17(6), pp. 1771-1782, 2007.

[22] Rozenfeld A. F., Arnaud-Haond S., Hernández-García E., Eguíluz V. M., Serrão E. A. \& Duarte C. M., Network analysis identifies weak and strong links in a metapopulation system. Proceedings of the National Academy of Sciences, 105(48), pp. 18824-18829, 2008.

[23] Borgstrom S. T., Elmqvist T., Angelstam P. \& Alfsen-Norodom C., Scale mismatches in management of urban landscapes. Ecology and Society, 11(2), 2006. 